\title{
An automated two-choice test of olfactory working memory in the rat: Effect of scopolamine
}

\author{
BOYER WINTERS, WILLIAM R. MATHESON, IAIN S. MCGREGOR, and RICHARD E. BROWN \\ Dalhousie University, Halifax, Nova Scotia, Canada
}

\begin{abstract}
Experimenters have often employed visual and/or spatial stimuli to assess rodent working memory in delayed (non)matching-to-sample or delayed response paradigms. We present an automated task that uses olfactory stimuli to test working memory in the rat. Rats were trained on a continuous delayed two-choice working memory task in an operant olfactometer with four different delays $(3,30,60$, and $120 \mathrm{sec}$ ). The rats required a mean of 12 sessions to achieve a criterion of $80 \%$ correct on two consecutive days with a 3-sec delay. Baseline data revealed a significant mnemonic function, with performance decreasing at the longer delays. Despite this significant effect, rats reached levels of approximately $76 \%$ correct at a retention interval (delay) of $120 \mathrm{sec}$. When a stable baseline level of performance was reached, the effect of the central muscarinic antagonist scopolamine hydrobromide was assessed. Scopolamine caused a dose-dependent, delay-independent decrease in performance. This result supports previous findings with scopolamine in other tests of rodent working memory and demonstrates the sensitivity of the two-choice olfactory task to this robust cognitive effect. A rodent working memory paradigm bearing closer resemblance to common human and nonhuman primate tests of working memory than do the often employed delayed response procedures [e.g., delayed (non)matching to position] may be highly valuable in the study of the biological substrates of short-term/working memory. The present experiment demonstrates the utility of the two-choice olfactory working memory task, which is sensitive to increasing delays and anti-cholinergic treatment and is rapidly acquired by rats.
\end{abstract}

The term working memory refers to the temporary storage and manipulation of information in cognitive tasks (Baddeley, 1986). In animal research, working memory is most often considered to involve the maintenance of trial-specific information for use "on line" or following relatively short delays (Olton, Becker, \& Handelmann, 1980). Impairments in working memory have been shown in aged humans and those suffering from senile dementia of the Alzheimer's type (Baddeley, 1986). Two commonly used methods for testing working memory, the delayed matching-to-sample (DMTS) and delayed nonmatching-to-sample (DNMTS) procedures, have been widely used to test object recognition memory in nonhuman primates (e.g., Elrod, Buccafusco, \& Jackson, 1988; Herzog, Grant, \& Roberts, 1977; Murray \& Mishkin, 1986, 1998) and are sensitive to amnesia in humans (e.g., Aggleton, Nicol, Huston, \& Fairbairn, 1988). In this paradigm, the subject is presented with a sample stimulus, followed after a delay by two choice stimuli, one that is the same as the sample and one that is different. The subject must choose the stimulus that matches (DMTS) or does not match (DNMTS) the sample in order to receive reinforcement. This paradigm eliminates the possibility of mediating responses, such as position habits or orienting responses, which are problematic for other (particularly

Correspondence should be addressed to R. E. Brown, Department of Psychology, Dalhousie University, Halifax, NS B3H 4JI Canada (email: rebrown@is.dal.ca). automated) working memory procedures, such as the delayed (non)matching-to-position [D(N)MTP] procedure (Chudasama \& Muir, 1997; Herremans, Hijzen, Welborn, Olivier, \& Slangen, 1996; Stanhope, McLenachan, \& Dourish, 1995), a paradigm commonly employed in the rodent literature.

The $\mathrm{D}(\mathrm{N}) \mathrm{MTP}$ is a robust measure of working memory in the rat, yet it has several drawbacks. In addition to the potential for mediating strategies, the $D(N) M T P$ presents several interpretational ambiguities. Since it is a delayed response task (as opposed to a delayed comparison task), the $\mathrm{D}(\mathrm{N}) \mathrm{MTP}$ actually provides the animal with all of the information needed to respond correctly after the delay before the delay begins (Steckler, Drinkenburg, Sahgal, \& Aggleton, 1998). For this reason, it cannot be said with absolute certainty that the $D(N) M T P$ measures recognition memory and not recall. Furthermore, the D(N)MTP uses spatial cues (i.e., the side on which the sample lever was presented) to guide performance, and it is possible for rats to perform successfully using either an allocentric or an egocentric strategy. These concerns may not be directly relevant for some experimenters' purposes; however, for those interested in the study of specific cognitive processes, more easily interpretable tasks are required. In light of these concerns, rodent studies should endeavor to use tasks that bear closer resemblance to those used most commonly in human and nonhuman primate studies of short-term/working memory. Unfortunately, experimenters have had little success in the past in training rats to perform at levels above chance in 
delayed versions of matching- or nonmatching-to-sample tasks (Steckler et al., 1998). One paradigm, however, which closely resembles the D(N)MTS task is the continuous nonmatching-to-sample (CNM) task of Pontecorvo (1983). In the CNM task, a stimulus serves as both the choice stimulus for the previous trial and the sample stimulus for the current trial. A stimulus is presented, followed by a variable delay, and then a second stimulus is presented, which can be either the same as (match) or different from (nonmatch) the first stimulus. Rats are typically required to respond at one of two locations, depending on whether the trial is a match or a nonmatch trial. Rats have been shown to perform in the CNM task at levels of $80 \%-90 \%$ correct, with retention intervals of up to $20 \mathrm{sec}$ (Pontecorvo, 1983; Pontecorvo, Clissold, White, \& Ferkany, 1991; Spencer, Pontecorvo, \& Heise, 1985). Since rats can perform this task relatively successfully, and since the CNM task lacks many of the ambiguities of the D(N)MTP task, it may be a more appropriate paradigm for the analysis of the specifics of rodent short-term/ working memory.

Short-term olfactory memory in the rat may be analogous to some forms of primate visual working memory (Slotnick, 1994), and such a model could provide a reliable, efficient means for the investigation of working memory processes that might be more easily compared with findings from human and monkey studies employing visual stimuli. Rats' ability to solve olfactory problems is well documented. Rats perform better in oddity problems with olfactory cues than they do in similar tasks with visual cues (Langworthy \& Jennings, 1972). Rats readily learn a series of discrimination problems, and the progressive improvement in their ability to solve these problems suggests the acquisition of a learning set (Jennings \& Keefer, 1969). In a series of 16 two-odor discrimination problems, rats rapidly acquired a learning set and showed one-trial learning by the end of the problem series (Slotnick \& Katz, 1974). Thus, when olfactory stimuli are used, rats can show learning abilities similar to those usually demonstrated only by primates in visual tasks (e.g., Harlow, 1949). Slotnick, Kufera, and Silberberg (1991) found comparable results with rats trained on more difficult discriminations, involving eight different odors. Nigrosh, Slotnick, and Nevin (1975) further demonstrated the importance of stimulus modality by showing that rats were successful in discrimination reversal problems when odors, but not lights or tones, were used as stimuli.

Several paradigms have been used with rats to assess working memory in the visual and auditory modalities with retention delays ranging from 1 to $120 \mathrm{sec}$ (Lyford, Gutnikov, Clark, \& Rawlins, 1993; Pontecorvo, 1983; Pontecorvo et al., 1991; Rothblat \& Hayes, 1987; Sakurai \& Wenk, 1990; Spencer et al., 1985; Viscardi \& Heise, 1986). However, working memory paradigms utilizing olfactory cues have been much less common. Ravel, Vigouroux, Elaagouby, and Gervais (1992) developed a DMTS task in which rats sampled an odor in one compartment of a T-maze and then chose the matching odor from two more odor sample boxes in another compartment. On the basis of this choice, the subject entered either the left arm or the right arm of the maze and received reinforcement. Rats performed at $85 \%$ correct with $4-$ or 30 -sec delays between the sample and choice odors, and there was a delaydependent decrease to $80 \%, 62 \%$, and $50 \%$ correct with delays of 60,120 , and $180 \mathrm{sec}$, respectively. Otto and Eichenbaum (1992) developed an odor-guided CNM task using a go-no-go procedure in an operant chamber. Rats trained on this task using a set of 16 odors with a 3sec delay between sample and choice odors obtained at least $80 \%$ correct. Lengthening the delay to 30 or $60 \mathrm{sec}$ significantly reduced the percent correct, as did reducing the number of odors in the odor set from 16 to 8,4 , or 2 . These two findings demonstrate the sensitivity of this task to delay and interference, two manipulations that affect performance in other kinds of memory tasks.

In the present study, we utilized an olfactory working memory task similar to that of Otto and Eichenbaum (1992), but which incorporates the "two-choice" design of the task used by Pontecorvo et al. (1991). The twochoice method provides many improvements over the typical go-no-go design often employed in rodent working memory tasks (e.g., Koger \& Mair, 1994; Zyzak, Otto, Eichenbaum, \& Gallagher, 1995). In the twochoice olfactory working memory task, reinforcement is available on every trial from one of two locations, depending on the condition (match or nonmatch). We feel that this is an improvement over the go-no-go paradigm for several reasons. First, the two-choice paradigm eliminates the ambiguity of correct rejections. Rather than inferring learning from a null response (a correct rejection, or no response on a trial on which reinforcement is not available), the two-choice design requires the animal to respond to both matching and nonmatching odors. This procedure, therefore, does not require the animal to inhibit responding to matching odors; rather, it requires the animal to respond at the correct spout for reinforcement. We feel that this is a better and more easily interpretable measure of the subjects' learning and memory because the animal must respond correctly on every trial or else forfeit reinforcement opportunities. In the go-no-go procedure, there is very little or no cost for a thirsty rat to have a "false alarm" (i.e., responding on a match trial), and this can inflate the error rate. Moreover, trials on which a rat fails to respond due to lack of attention or motivation will be counted in the go-no-go paradigm as errors of omission, perhaps falsely suggesting a mnemonic impairment. Finally, we feel that the two-choice paradigm is an improvement on the go-no-go paradigm because reinforcement is available on every trial. Because of this, and because subjects do not have to learn to inhibit responses on certain trials, their attention and motivation to learn the task may be increased. If subjects pay attention to the sample stimuli, they can receive reinforcement on every trial. This is not the case in the go-no-go paradigm, in which reinforcement is available on only half of the trials. 
In the present study, rats were trained to stable levels of baseline performance in the two-choice olfactory working memory task. Following the demonstration of delaydependent baseline performance, the muscarinic antagonist scopolamine $\mathrm{HBr}$, which is well known to produce delay-independent effects in other rodent working memory paradigms (e.g., Dunnett, Rogers, \& Jones, 1989; Herremans et al., 1996; Spencer et al., 1985), was administered. This was done to assess the sensitivity of the twochoice olfactory working memory task to the particularly robust cognitive effect of this anticholinergic drug.

\section{METHOD}

\section{Subjects}

The subjects were 5 male Long-Evans hooded rats (Rattus norvegicus), 120 days old, obtained from Charles River Canada Inc. (St. Constant, Quebec) at 90 days of age. Their mean weight at the start of the experiment was $340 \mathrm{~g}$. The rats were housed individually in $48 \times 27 \times 16 \mathrm{~cm}$ polycarbonate cages, with softwood chip bedding and stainless steel grid tops, in an environmentally controlled room on a 12:12-h light:dark cycle (lights off at 8 a.m.). Food (Ralston Purina Laboratory Rodent Chow) was available ad libitum; access to water was limited to a 10 -min period each day following training. All training was conducted during the dark phase of the light:dark cycle.

\section{Apparatus}

The apparatus consisted of two computer-controlled olfactometers connected to two separate operant chambers. The apparatus was housed in a single test room, which was kept dark during testing.

Olfactometers. Eight different odors could be delivered to the operant chambers through the computer-controlled olfactometers (see Figure 1). The design of these olfactometers was based on the four-odor olfactometer described by Schellinck, Brown, and Slotnick (1991). Clean air, which was provided by a continuous-duty
GAST compressor pump [(1) in Figure 1] and filtered through an activated charcoal filter (2) and two fritted glass filters (3), was delivered continuously to the test chamber through the open ports of a three-way solenoid final valve (General Valve Model 9-321-900) (10). This flow of clean air bypassed the stimulus channels when an odor was not being presented. The flow of air into each stimulus channel and the subsequent release of an odor stimulus from each stimulus tube (8) were controlled by the action of three two-way solenoid valves. Airflow from the stimulus channel flowmeter passed through a normally closed two-way valve (General Valve Model 1$30-900)(6)$ and then entered another two-way valve (General Valve Model 3-507-900) (7), upstream from the stimulus tube. The opening of this second valve allowed the air to enter the stimulus tube, becoming saturated with odorant. An identical two-way valve (9) then opened downstream from the stimulus tube, allowing this saturated air to mix with the main air stream; this mixture was then sent to the odor sampling port (11) through the normally open port of the three-way final valve. On the completion of a proper nosepoke in the odor sampling port, the normally open port of the final valve closed briefly, and the remaining odorized air was diverted through the normally closed port of the final valve to an exhaust line. This air was exhausted through a ceiling vent. The clean airstream was monitored by the main air flowmeter (Model B-125-50) (4), and the flow of air to the stimulus channels was monitored by a second flowmeter (Model A-125-7) (5).

Operant chambers. All training occurred in two identical 22.25 $\times 27 \times 17 \mathrm{~cm}$ Plexiglas operant chambers mounted on a wall of the test room, with an access door in the side opposite the wall. Each rat was trained in the same chamber in all sessions. An odor sampling port with a diameter of $2 \mathrm{~cm}$ was centered on the right wall of the chamber $12.5 \mathrm{~cm}$ above the floor. Each chamber contained two water spouts, which delivered reinforcement, one $5 \mathrm{~cm}$ on each side of the odor sampling port at the same height as the sampling port. Each of these water spouts was connected by a tube to a $10-\mathrm{ml}$ reservoir. Responses to the odor sampling port were monitored by a photoelectric cell. When the rat poked its nose into the port, this broke a photoelectric beam, and the duration of this nosepoke response was recorded. Responses to the water spout were detected

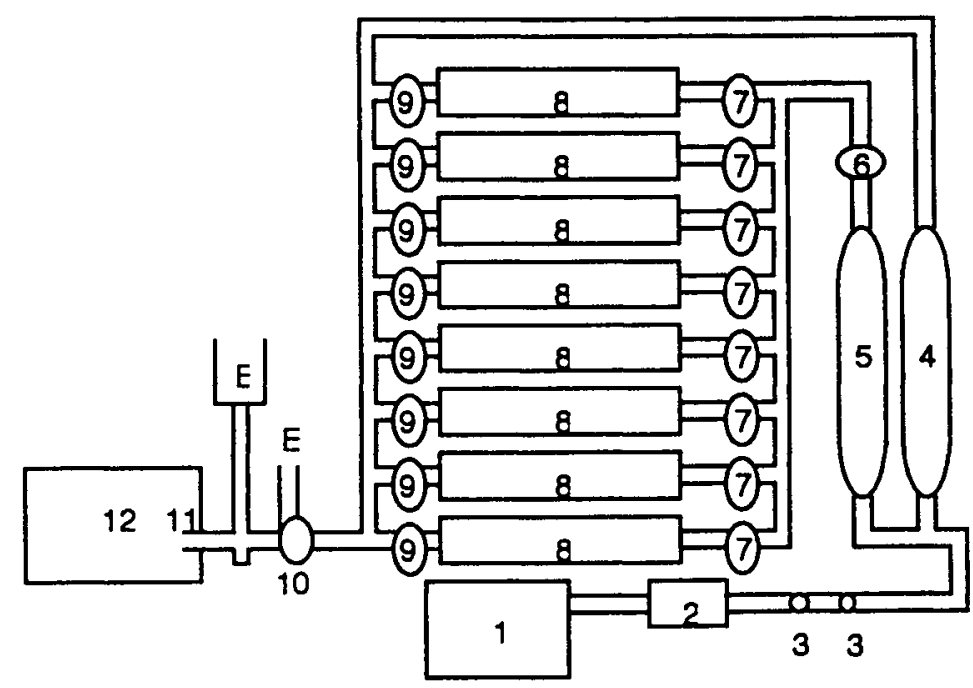

Figure 1. A schematic representation of the olfactometer. (1) GAST compressor pump, (2) activated charcoal filter, (3) fritted glass filters, (4) main air flowmeter, (5) stimulus channel flowmeter, (6) two-way solenoid valve, (7) twoway valve upstream from stimulus tube, (8) stimulus tubes, (9) two-way valve downstream from stimulus tube, $(10)$ final valve, (11) odor sampling port, (12) operant chamber, $E=$ to exhaust. 
and recorded electrically as the rat completed an electric circuit between the spout and the floor each time it made contact with the spout. To ensure that the rats were conductive, glycerin was rubbed onto the right hind foot of each subject prior to testing in the operant chamber. Correct responses were reinforced by the delivery of $0.1 \mathrm{ml}$ of water from a reservoir controlled by a two-way solenoid valve (General Valve Model 2-40-900). A 24-V houselight, which signaled the start of a trial, was mounted $5 \mathrm{~cm}$ behind the left wall of the chamber at the height of the ceiling of the chamber. A buzzer, through which auditory feedback regarding proper nosepoke duration could be provided to each subject, was located below each chamber. The auditory signal differed between the two chambers: In Chamber 1, the signal was a "beep," whereas in Chamber 2, it was a "buzz." All sessions were controlled and recorded by Strawberry Tree's Workbench program, running on a Macintosh IIci computer (McGregor, 1996).

\section{Odors}

The eight odors used were patchouli oil $(20 \%$ in diethyl phthalate, DEP), star anise oil ( $10 \%$ in DEP), grass (cis-3-hexenol, $2 \%$ in DEP), pepper oil ( $10 \%$ in DEP), castoreum resin artessence ( $2 \%$ in DEP), wintergreen (methyl salicylate, $10 \%$ in DEP), amyl acetate ( $5 \%$ in DEP), and jasmine ( $50 \%$ in DEP). These odors were generously donated by Givaudan Roure Corporation (Teaneck, NJ) and were described by the supplier as being of equal intensity as determined by a human testing panel.

\section{Procedure}

The experimental procedure consisted of four phases: shaping, task training, delay training, and testing with scopolamine.

Shaping. Three shaping sessions were completed. The first of these sessions trained the subjects to receive reinforcement from the water spouts with no nosepoke requirement. Early trials involved some hand-shaping to start the subjects licking for reinforcement. Regular trials began after the subjects had been handshaped to drink from the spouts. The beginning of each trial was signaled by the illumination of the houselight, indicating the availability of reinforcement. The reinforced water spout varied from left to right pseudorandomly over trials. Over the 96 trials, each spout was reinforced an equal number of times. The subjects were not penalized for incorrect responses. If the subject responded initially to the wrong spout, the trial continued until the correct spout was chosen. Therefore, the session did not end until the subject had received a total of 96 reinforcements, 48 at each spout. A response to the correct spout was accompanied by a brief auditory signal, followed by the extinction of the houselight and a $3-\mathrm{sec}$ delay before the start of the next trial.

The final two shaping sessions trained the subjects to nosepoke for a certain duration in order to be eligible for water reinforcement. The subjects were initially hand-shaped to poke their noses into the odor sampling port; when a nosepoke of the correct duration was made, an auditory signal sounded, and water was available at one of the two spouts. The correct spout was determined by the odor sequence. All eight odors were used and presented in a pseudorandom sequence of matches and nonmatches. On a match trial, when the current odor was the same as the previous odor, the correct response was at the left spout. On nonmatch trials, in which the current odor differed from the previous odor, the correct response was at the right spout. A correction procedure was in place whereby, if an incorrect response was made, the subjects were given a 20 -sec period in which they could respond to the correct spout. During the second shaping session, the rats were initially required to nosepoke for $0.2 \mathrm{sec}$ before the final valve opened, delivering odor to the sample port. The rats were then required to poke for an additional $0.2 \mathrm{sec}$ before being eligible for reinforcement. The poking requirement increased gradually, such that, by the end of the session, the rats were required to poke for an initial $1 \mathrm{sec}$ before odor was delivered and a minimum of an additional $0.2 \mathrm{sec}$ before being eligible for reinforcement. If the rats failed to nosepoke for the minimum $0.2 \mathrm{sec}$ of odor presentation (a "short sample"), then odor delivery was discontinued after $1 \mathrm{sec}$, and the rat had to repeat the initial $1-\mathrm{sec}$ nosepoke before again being presented with the sample stimulus. A total of 192 trials were run, with a $3-\mathrm{sec}$ delay between odor presentations.

The third and final shaping session was identical to Session $2 \mathrm{ex}-$ cept that the rats initially had to nosepoke for $0.4 \mathrm{sec}$ before odor delivery commenced and an additional $0.2 \mathrm{sec}$ before reinforcement became available. As in Session 2, the initial nosepoke requirement gradually increased throughout the session so that the final requirement of $1.2 \mathrm{sec}$ was in place by the end of the session.

Task training. Following shaping, the subjects were trained to go either left or right depending on whether the trial was a match or nonmatch trial. Reinforcement was available only at the right-hand spout on nonmatch trials and was available only at the left spout on match trials. If the correct response was made, water was delivered from the appropriate spout, the houselight was extinguished, and a 3 -sec delay occurred before the next odor presentation. If an incorrect response was made, the trial ended, extinguishing the houselight and initiating a $3-\mathrm{sec}$ delay before the next odor presentation. If no response was made by the rat at either spout within $5 \mathrm{sec}$ of sampling the odor, the trial ended and was recorded as an omission, initiating the 3 -sec delay before the next odor presentation. In the case of an omission, the previous sample odor was used for the next trial. To ensure that the association between type of trial (match or nonmatch) and correct response (lick left or right) was made, these training sessions consisted of blocks of match and nonmatch trials. The first 100 trials consisted of alternating blocks of 10 match (go left) and 10 nonmatch (go right) trials. Trials 101-155 consisted of alternating blocks of 5 match trials followed by 5 nonmatch trials. The final 37 trials were alternating blocks of 3 match trials followed by 3 nonmatch trials.

Delay training. Once the subjects reached a criterion of $80 \%$ correct for 2 consecutive days on the blocked task training sessions, pseudorandomized trials were introduced, the number of trials was reduced from 192 to 96 per session, and four different delays were gradually incorporated into the task. Initially, the subjects were run on the task with a pseudorandomized sequence of matches and nonmatches using only the $3-\mathrm{sec}$ delay between odor presentations. A criterion of $80 \%$ correct for 2 consecutive days was used for each step of this phase of training. Once this criterion was reached on the 3-sec task, a 30-sec delay was introduced in addition to the 3-sec delay, resulting in a session with a randomized sequence in which half of the trials had 3-sec delays and half had 30-sec delays. When criterion performance was reached with two delays, a $60-\mathrm{sec}$ delay was added so that each of the three delays was utilized for one third of the 96 trials. Finally, when criterion performance was reached with three delays, the fourth delay, $120 \mathrm{sec}$, was introduced. Thus, the multiple-delay two-choice task consisted of a session of 96 trials, utilizing four delays, each in effect for approximately one quarter of the trials. The final nosepoke requirement was $1.2 \mathrm{sec}$. That is, a nosepoke following the end of the retention interval resulted in the opening of the odor valves of the appropriate stimulus tube for $2 \mathrm{sec}$. However, for the first half of this period, the final valve was switched to the exhaust, such that no odor was yet available to the rat. At the end of this first second of nosepoking, the final valve was switched to allow odor to flow into the odor sampling port. The rats were then required to make at least one nosepoke of $0.2 \mathrm{sec}$ or greater during the last second of odor presentation in order to be eligible for reinforcement. Failure to nosepoke for at least $0.2 \mathrm{sec}$ meant that the rat had to repeat the initial $1-\mathrm{sec}$ nosepoke duration before the next odor presentation - that is, a short sample lead to the reinstatement of the total $1.2-\mathrm{sec}$ nosepoke requirement. There was no scheduled limited hold period, and omissions were never observed during the testing phase of the experiment. The subjects consistently completed each 96-trial session in 90-100 min. Following 
an incorrect response, the houselight was extinguished, and the next scheduled retention interval commenced immediately. Incorrect trials were not repeated. Each of the eight odors was presented with approximately equal frequency, and each session consisted of approximately equal numbers of matching and nonmatching trials.

Testing with scopolamine. When the subjects achieved a stable level of baseline performance, drug testing was initiated using the muscarinic antagonists scopolamine $\operatorname{HBr}(0.03,0.1$, and $0.3 \mathrm{mg} / \mathrm{kg}$; Sigma) and scopolamine $\mathrm{MeBr}(0.3 \mathrm{mg} / \mathrm{kg}$; Sigma $)$, which was used as an active control for possible peripheral effects of scopolamine. Saline was also given as a control. Each rat received a single injection of each of the five treatment conditions. Administration was determined by a modified Latin square design to control for potential order effects, and each treatment day was followed by
3 "recovery days" on which the rats were run without injections. This resulted in a total of 17 testing days. For example, Day 1 was the first injection day, Days 2-4 were recovery days, Day 5 was the second injection day, and so on. This was done to ensure that the subjects returned to baseline performance before the next drug testing day. All injections were given i.p., $30 \mathrm{~min}$ prior to testing.

\section{RESULTS}

\section{Task Training}

The rats required a mean of 12 sessions (range $=6-16$ ) of task training to reach the criterion of $80 \%$ correct for two consecutive sessions.
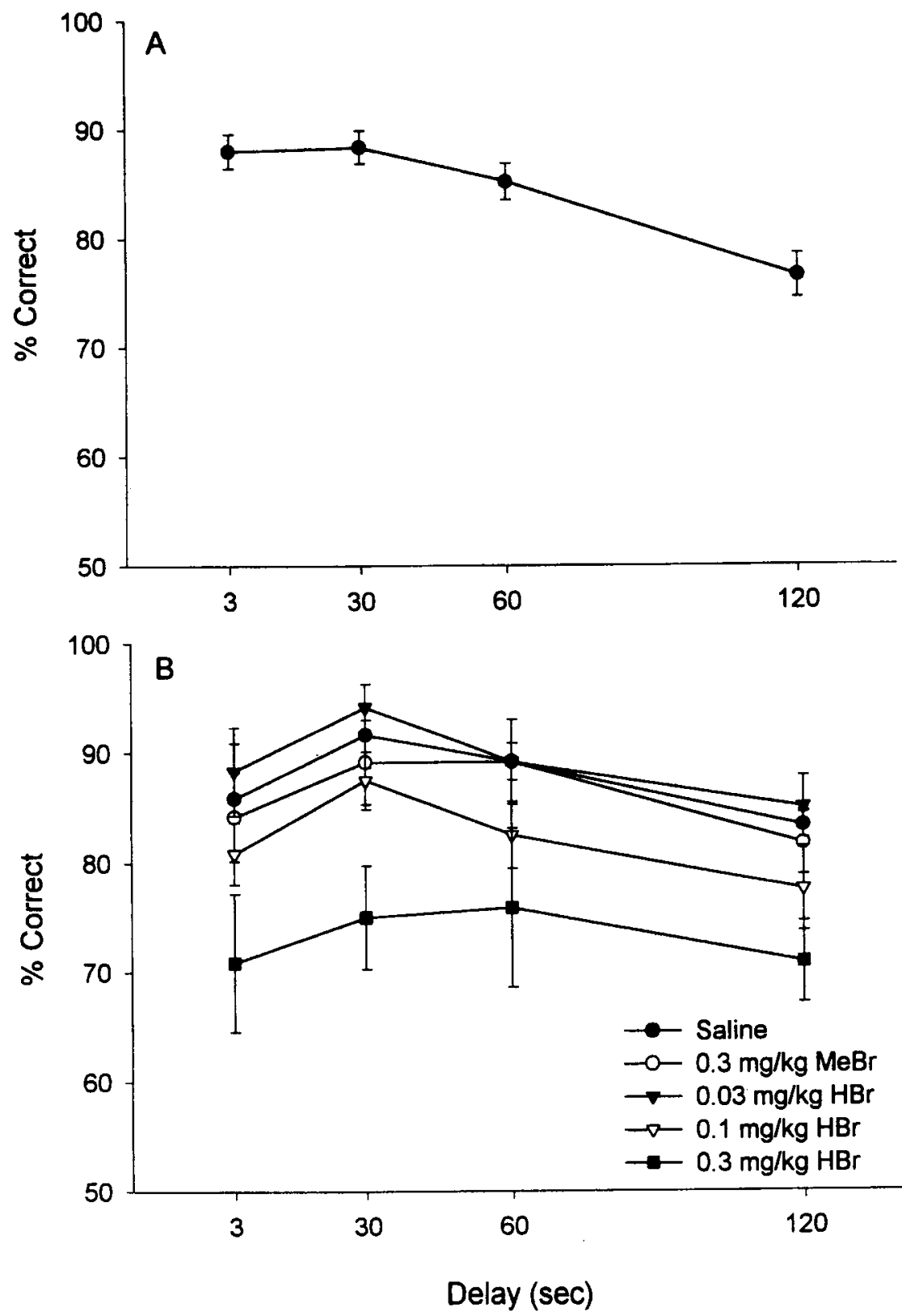

Figure 2. The mean percent correct $( \pm S E)$ at each delay from $(A)$ the five sessions prior to scopolamine testing and $(B)$ the five injection sessions of the scopolamine testing phase. 


\section{Delay Training}

The rats were run for a mean of 27 sessions (range $=$ 23-34) on the multiple-delay version of the task before drug testing began. Figure $2 \mathrm{~A}$ shows the overall percent correct for the 5 rats from performance on the last five sessions of delay training prior to the beginning of the scopolamine sessions. A delay-dependent memory function is illustrated with the percent correct decreasing from about $88 \%$ at the 3 - and 30 -sec delays to $85.17 \%$ at $60 \mathrm{sec}$ and $76.50 \%$ at $120 \mathrm{sec}$. A one-way repeated measures analysis of variance (ANOVA) revealed a significant effect of delay $[F(3,12)=36.07, p<.001]$, and multiple orthogonal post hoc comparisons showed significantly better performance at $60 \mathrm{sec}$ than at $120 \mathrm{sec}[F(1,3)=44.53$, $p<.001]$ and significantly better performance at 3 and $30 \mathrm{sec}$ than at 60 and $120 \mathrm{sec}$ combined $[F(1,3)=63.62$, $p<.001$, but no significant difference between performance at $3 \mathrm{sec}$ and $30 \mathrm{sec}[F(1,3)<1.0$, n.s. $]$.

\section{Scopolamine Test}

The percent correct for each drug condition across the four delays is presented in Figure 2B. A two-way ANOVA with repeated measures indicated a significant main effect of drug $[F(4,16)=8.03, p<.001]$ and a significant main effect of retention interval $[F(3,12)=9.05, p<.005]$, but the interaction between drug and retention interval was not significant $[F(12,48)<1.0$, n.s. $]$. Figure $2 \mathrm{~B}$ indicates that increasing the dose of scopolamine $\mathrm{HBr}$ caused a similar reduction in percent correct across all delays. Multiple post hoc comparisons revealed that the percent correct at the highest dose $(0.3 \mathrm{mg} / \mathrm{kg})$ of scopolamine $\mathrm{HBr}$ was significantly less than at the lowest dose $(0.03 \mathrm{mg} / \mathrm{kg})$ $[F(1,4)=25.20, p<.001]$. In addition, performance was significantly lower at the highest dose of scopolamine $\mathrm{HBr}$ than it was with scopolamine $\operatorname{MeBr}[F(1,4)=16.33, p<$ $.001]$. The overall percent correct of the combined scopolamine $\mathrm{HBr}$ doses was also significantly lower than that of saline $[F(1,4)=5.35, p<.05]$.

Figure 2B also illustrates the main effect of delay. Generally, there was a consistent decrease in percent correct as delay increased from 30 to 60 to $120 \mathrm{sec}$. The low performance at $3 \mathrm{sec}$, relative to the other delays, was not expected. The subjects performed considerably worse at 3 -sec delays than at either 30- or 60-sec delays but better than at $120 \mathrm{sec}$ delays. This effect was reflected in multiple orthogonal post hoc comparisons, which revealed a significant quadratic effect of delay. Performance at the two intermediate delays ( 30 and $60 \mathrm{sec}$ ) was significantly higher than performance at the 3- and 120-sec delays $[F(1,3)=23.01, p<.001]$.

To assess the effect of recovery days (i.e., sessions separating drug days, in which the rats were run without injections), we also looked at the mean differences in percent correct between drug days and the preceding recovery day. A two-way repeated measures ANOVA indicated a significant main effect of drug when performance on the treatment day was compared with performance on the previous, nondrug, day $[F(4,16)=3.85, p<.05$, data not shown]. Multiple orthogonal post hoc comparisons showed that this was due to a significant decrease from previous recovery day performance caused by the highest dose of scopolamine $\mathrm{HBr}$ relative to all other treatment conditions $[F(1,4)=13.11, p<.01]$, which did not differ from one another.

\section{Match Versus Nonmatch Trial Performance}

Figure 3A shows the percentage of the total number of incorrect responses at each delay, which is accounted for by match and nonmatch trials over the 5 days prior to scopolamine testing. A two-way repeated measures ANOVA failed to reveal significant effects of trial type $[F(1,4)=$ $4.37, \mathrm{n} . \mathrm{s}$. $]$ or delay $[F(3,12)=0, \mathrm{n} . \mathrm{s}$.], but there was a trend toward a significant delay $\times$ trial type interaction $[F(3,12)=2.92, p=.078]$. Figure $3 \mathrm{~A}$ reveals that this was due to an increase in the percentage of incorrect responses at $120 \mathrm{sec}$ being accounted for by match trials. Match trials constituted $80 \%$ of the total number of incorrect responses at $120 \mathrm{sec}$.

Figure 3B illustrates the percentage of the total number of incorrect responses at each delay by trial type over the 5 injection days of the scopolamine testing phase of the experiment. The main effects of trial type $[F(1,4)=$ 5.00$, n.s. $]$ and delay $[F(3,12)=1.00$, n.s. $]$ were not significant; however there was a significant delay $\times$ trial type interaction $[F(3,12), p<.02]$. A post hoc comparison using the Tukey test revealed that performance was significantly worse on match trials than on nonmatch trials at delays of $3 \sec (p<.01)$ and $120 \sec (p<.05)$. Match trials accounted for approximately $75 \%$ and $67 \%$ of the total number of incorrect responses at 3-and 120-sec delays, respectively. Performance did not differ significantly by trial type at delays of $30 \sec (p>.05)$ or $60 \sec (p>.05)$.

\section{DISCUSSION}

The two-choice olfactory working memory task presented in the present experiment provides a relatively unambiguous measure of short-term recognition memory in the rat. Certain maze paradigms (e.g., the radial arm maze) and operant delayed response procedures [e.g., D(N)MTP], while obviously requiring the use of some form of working memory, are difficult to describe in terms of the specific processes involved. For example, delayed response tasks, by providing all of the relevant information prior to the actual delay, might actually assess recall rather than recognition memory (Steckler et al., 1998). This is not a major drawback when one is merely interested in short-term memory effects per se. However, in order to fully investigate the important subtleties of cognitive processes, it is necessary to utilize less ambiguous tests. By employing the CNM paradigm of Pontecorvo (1983) in combination with olfactory cues, we have presented such an unambiguous test of short-term recognition memory in the rat, which may also result in better rates of acquisition and higher levels of performance due to the increased salience of the olfactory stimuli used. 

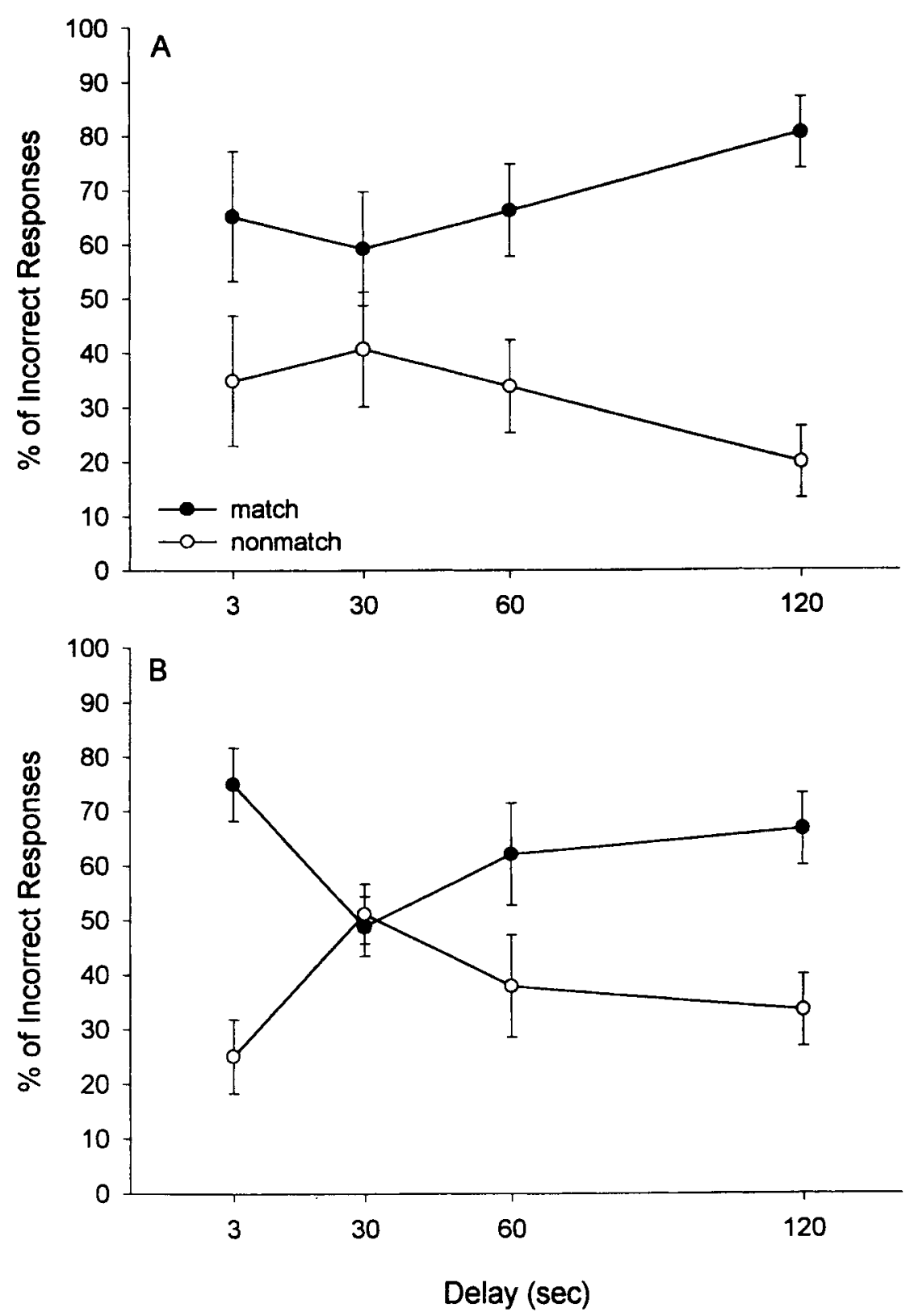

Figure 3. The mean percentage $( \pm S E)$ of the total number of incorrect responses at each delay by trial type (match or nonmatch) from (A) the five sessions prior to scopolamine testing and $(B)$ the five injection sessions of the scopolamine testing phase.

The CNM paradigm, much like a true $\mathrm{D}(\mathrm{N}) \mathrm{MTS}$ design, requires the subject to retain a representation of the initial sample stimulus over a variable delay period. The subject must then use this representation to guide behavior in response to the choice stimulus presentation following the delay. Thus, the CNM paradigm can be said to be a relatively clear test of short-term recognition memory.

The use of olfactory stimuli in a CNM design provides what may be a more appropriate assessment of short-term/ working memory in the rat. There is a substantial literature detailing the successful performance of rats in olfactory- guided learning and memory paradigms (Slotnick, 1994). It is apparent from this literature that rats may be capable of much higher levels of performance in tasks with olfactory cues than in similar tasks using visual or even auditory stimuli. The present study adds further support to this assertion by demonstrating the ease with which rats can acquire the two-choice olfactory task with a short retention interval $(3 \mathrm{sec})$. Rats reached criterion levels of performance with a 3 -sec delay in a mean of 12 sessions. Moreover, the present experiment provides insight into the relatively large short-term memory span of rats when 
tested with olfactory stimuli. Baseline performance (see Figure $2 \mathrm{~A}$ ) revealed that, despite a significant delaydependent decline in performance, rats reached a mean level of approximately $76 \%$ correct with a retention interval of $120 \mathrm{sec}$. Otto and Eichenbaum (1992) similarly found performance of rats in their go-no-go olfactory task to be between $75 \%$ and $80 \%$ correct with a delay of $60 \mathrm{sec}$ and a stimulus set of 16 odors.

Pontecorvo and colleagues have often employed trial parameters in the CNM task that lead to an uneven ratio of match to nonmatch trials (Pontecorvo, Sahgal, \& Steckler, 1996). The rationale for this approach has been that using approximately three times as many match as nonmatch trials would increase the salience of the latter, leading to increased rates of acquisition. However, the present experiment demonstrates that a continuous nonmatching task using a 1:1 ratio of match and nonmatch trials results in similarly rapid acquisition and subsequent high levels of performance. This finding is important, since an unbalanced ratio of match and nonmatch trials could potentially lead to the acquisition of a response bias during training (Pontecorvo et al., 1996). The demonstration of rapid acquisition with a balanced trial ratio suggests that training animals with an uneven ratio is unnecessary.

One unexpected finding of the present experiment involves the relatively low performance of rats at the 3 -sec delay. It is not possible that the 3-sec delay provided insufficient time for water consumption, since this act required only a fraction of a second, and the trial did not progress until a response had been completed at one of the water spouts. Thus, the progression of each trial was under the rat's control. Following a stimulus presentation, the rat responded at one of the two water spouts (errors of omission were never observed). A water spout response extinguished the houselight and initiated the next retention interval. Thus, in this sense, the rat always had sufficient time to process the sample stimulus and respond at a water spout. It is possible that the large number of events occurring within such a relatively short period of time (i.e., nosepoke response, match/nonmatch decision, water spout response, houselight off, 3-sec delay, houselight on) may have caused some kind of attentional deficit whereby the rats were unable to completely (or sufficiently) process the sample stimulus at the 3-sec delay. However, Pontecorvo et al. (1991) found no such deficit at the shortest delay $(2.5 \mathrm{sec})$ in a similarly designed CNM task with tones and lights as stimuli.

One possible explanation of this result is that the olfactory representation of the sample stimulus interfered with the incoming choice stimulus on trials with short delays, leading to confusion during the choice phase of the task. This could occur if, for instance, the 3-sec delay allowed insufficient time for the sample olfactory "trace" to pass from an immediate or "sensory" type of memory store to a short-term store. Such a "leftover" sensory memory might interfere with an incoming olfactory stimulus during the choice phase. However, an analysis of the mean actual delays from the testing phase of this experiment reveals that the shortest delay actually averaged $13.84 \mathrm{sec}(S E M= \pm 1.26)$ rather than $3 \mathrm{sec}$. This length of delay would be expected to provide sufficient time for the transfer of the sample representation from an immediate (sensory) memory to a short-term (working) memory store. Moreover, Otto and Eichenbaum (1992) reported no such deficit in their go-no-go version of the olfactory CNM task with a stimulus set of eight odors.

The finding that rats did not respond immediately following the delay in the " $3-\mathrm{sec}$ " condition may lend credence to the suggestion that some kind of attentional interference occurred during the choice phase at the shortest delay. The overall mean "actual delay" values (i.e., the time from when the rat made its last choice response until it made its next nosepoke response following a delay) by delay were $13.84 \mathrm{sec}( \pm 1.26 \mathrm{sec}), 38.11 \mathrm{sec}( \pm 0.99 \mathrm{sec})$, $67.60 \mathrm{sec}( \pm 0.59 \mathrm{sec})$, and $127.91 \mathrm{sec}( \pm 1.40 \mathrm{sec})$ for the $3-, 30-, 60-$, and $120-\mathrm{sec}$ delay conditions, respectively. The greater "lag" between the end of the delay and the next nosepoke in the 3-sec delay condition may be indicative of an attentional disruption under this particular testing condition. Whatever the implication, such a finding highlights the importance of using a limited hold procedure in future studies to better control the length of the intended retention interval.

It is possible that the rats in the present study were affected more at the shortest delay by interference from previous trials (proactive interference, PI). It is known that PI effects depend on two factors: intertrial agreement and intertrial interval (Edhouse \& White, 1988). Sample stimuli from previous trials that differ from the current sample can interfere with current trial performance, and this interference is more pronounced with shorter intertrial intervals. However, in a continuous task such as the CNM task, the intertrial interval and the retention interval (delay) are equivalent. Thus, a shorter retention interval might be expected to yield a higher degree of PI following nonmatch trials. This may have been the case in the present experiment, resulting in poorer performance at the shortest retention interval relative to the intermediate delays. However, it is worth noting that Pontecorvo (1983), in an extensive analysis of PI effects in the CNM task, found no such result at shorter retention intervals $(2.5 \mathrm{sec})$.

Thus, it remains unclear what caused the curious result at the shortest delay interval in the present experiment. The previous discussion, however, raises some important issues regarding the choice to use a continuous task in this study. One drawback of the CNM paradigm is the aforementioned equivalence in the task of retention delay and intertrial interval. This can lead to problems when trying to interpret results in terms of PI effects, as well as when trying to distinguish purely mnemonic from non-mnemonic effects in drug and lesion studies. The ability to vary the value of the intertrial interval in a (discrete) paired-trials task provides the advantage of direct study of the effects of PI on working memory. This 
is not possible in the continuous-trials version of the twochoice olfactory task. We are currently developing a pairedtrials version of this task for use in future studies. Not only will this facilitate interpretation of results, such as the shortest delay anomaly in the present study, but a pairedtrials procedure will also provide the ability to incorporate a condition that is much closer to an "immediate" delay. Such a condition is important in experiments investigating the effects of drugs or lesions on working memory because it provides a measure of task performance under conditions of relatively minimal mnemonic requirements (Pontecorvo et al., 1996). Results can be more easily interpreted in terms of mnemonic and nonmnemonic effects when such an immediate delay is used, since a disruption of performance under such conditions would be indicative of a non-mnemonic (e.g., attentional, motivational) deficit. An immediate delay cannot be employed in a continuous task, since the animal must be given sufficient time to respond for reinforcement following each stimulus presentation. Thus, a certain minimum delay must be allowed. Pontecorvo and colleagues have consequently often utilized a minimum delay of $2.5 \mathrm{sec}$ in their CNM experiments. Such a short delay should represent a relatively low memory requirement, yet it would be preferable to test animals at a 0 -delay condition. Hence, we feel that a paired-trials version of the two-choice olfactory working memory task described in the present experiment may provide optimal conditions for the study and analysis of the biological basis of working and short-term memory in the rat.

The analysis of performance by trial type suggests that the differential influence of match and nonmatch trials on performance is related to the difficulty of the task. This is best demonstrated by a comparison between the graphs in Figures 2 and 3. Figure 2A illustrates the good performance of rats at the three lowest delays relative to performance at $120 \mathrm{sec}$ in the five sessions prior to testing. This delay function was associated with the responding pattern shown in Figure 3A, wherein $80 \%$ of the incorrect responses at $120 \mathrm{sec}$ were made on match trials. Notably, performance at $3 \mathrm{sec}$ was better than or equal to that at 30 and $60 \mathrm{sec}$ in Figure $2 \mathrm{~A}$, and Figure $3 \mathrm{~A}$ illustrates the similarity in performance by trial type at these three delays relative to that at $120 \mathrm{sec}$. Conversely, in the testing sessions (see Figures $2 \mathrm{~B}$ and $3 \mathrm{~B}$ ), there was a significant quadratic effect of delay on percent correct responses, with rats performing significantly worse at 3 and $120 \mathrm{sec}$ than at 30 and $60 \mathrm{sec}$ (Figure 2A). This pattern of results was accompanied by a significant interaction between delay and trial type (see Figure 3A), in which match trials accounted for $75 \%$ and $67 \%$ of the total number of incorrect responses at the 3 - and $120-\mathrm{sec}$ delays, respectively. There were no such effects of trial type on performance at the 30- and 60-sec delays.

Thus, the overall effects of delay on performance tend to be reflected in the influence of trial type (match or nonmatch) on performance. This may indicate the use of alternate response strategies by rats as, for whatever rea- son, the task becomes more difficult. The above results may reflect one of two possible alternative strategies in the present experiment. First, since response location was not counterbalanced between rats (i.e., a nonmatch always required a response on the right side), these data may reflect a side bias. Rats, under cognitively taxing conditions, may have chosen to always respond at the right water spout. This would result in an increase in the percentage of incorrect responses occurring on match trials, since the correct response on such a trial was always at the left water spout. However, it seems unlikely that all subjects would choose to respond on the same side, since rats are known to show distinctive individual side biases under such conditions in other operant working memory tasks (e.g., Blokland \& Dunnett, 1995). An alternative explanation for the data is that rats chose to treat "uncertain" trials as nonmatching trials under conditions in which cognitive performance was taxed. Such a "cognitive" bias would guarantee levels of reinforcement of at least $50 \%$ on such uncertain trials.

The results from the scopolamine testing sessions generally support past findings with scopolamine, which suggest a non-mnemonic effect of the drug (Dunnett et al., 1989; Herremans et al., 1996; Spencer et al., 1985). Much recent evidence has implicated the cholinergic system in attentional processes (e.g., Jones \& Higgins, 1995; Muir, Everitt, \& Robbins, 1994; Voytko et al., 1994), and it is likely that scopolamine affected attention in the present experiment, thereby resulting in a delay-independent reduction in task performance. Despite some suggestion that scopolamine may impair olfaction (e.g., Serby et al., 1989; Soffie \& Lamberty, 1988), this is unlikely to have been the case since scopolamine methylbromide, a peripherally acting cholinergic antagonist, given at a dose of $0.3 \mathrm{mg} / \mathrm{kg}$, did not significantly reduce performance. If the negative effect of scopolamine $\mathrm{HBr}$ in this study was a result of an impairment of olfactory receptivity, then the peripherally acting scopolamine $\mathrm{MeBr}$ should have produced a decrement in percent correct similar to that produced by the highest doses of scopolamine $\mathrm{HBr}$. However, it is possible that systemic scopolamine $\mathrm{HBr}$ affects the function of neurons in the brain that are important for odor perception. It seems more likely, however, that systemic injection of the muscarinic antagonist scopolamine $\mathrm{HBr}$ resulted in attentional impairment in the two-choice olfactory working memory task. It is possible that systemically administered scopolamine has its most significant action within the central nervous system at the terminal regions of the cholinergic cells of the nucleus basalis magnocellularis ( $\mathrm{nbm}$ ). Recent evidence has indicated that the cholinergic nbm may play an important mediating role in attentional processes in the rat with little or no direct role in working memory retention per se (Everitt \& Robbins, 1997). The attentional impairment induced by systemic scopolamine does not necessarily preclude a role for acetylcholine in working memory retention; recent studies have suggested the involvement of the cholinergic septo-hippocampal pathway in such 
processes (Ohno, Yamamoto, \& Watanabe, 1994; Walsh, Herzog, Gandhi, Stackman, \& Wiley, 1996). It may be that systemic scopolamine exerts a greater effect in the terminal regions of the nbm than it does in the hippocampus and related terminal zones of the cholinergic projections from the medial septum.

Obviously, more specific methods will be required to elucidate the roles of the various cholinergic pathways in working memory. What the present study demonstrates is a potentially useful new task to facilitate this area of analysis. By eliminating several ambiguities of more commonly used tests of working memory, the two-choice olfactory working memory task provides a more easily interpretable measure of short-term/working memory recognition performance in the rat. Additionally, the use of odor cues rather than visual or auditory stimuli may result in quicker acquisition and better overall performance at longer delays, permitting more subtle investigation of the mechanisms of short-term memory retention. The two-choice olfactory working memory task is sensitive to anticholinergic treatment, yielding results similar to those found with scopolamine in more common paradigms, but with the advantage of greater ease of interpretation. The use of such a task in future studies may help to clarify the biological bases of short-term and working memory in the rat.

\section{REFERENCES}

Aggleton, J. P., Nicol, R. M., Huston, A. E., \& Fairbairn, A. F. (1988). The performance of amnesic subjects on tests of experimental amnesia in animals: Delayed matching-to-sample and concurrent learning. Neuropsychologia, 26, 265-272.

BADDELEY, A. (1986). Working memory. New York: Oxford University Press.

Blokland, A., \& DunnetT, S. B. (1995). Spontaneous response tendencies in noncontingent trials of a matching-to-position task in rats: Consequences for learning the matching and nonmatching task contingencies. Psychobiology, 23, 76-84.

Chudasama, Y., \& MuIR, J. L. (1997). A behavioural analysis of the delayed nonmatching to position task: The effects of scopolamine, lesions of the fornix and of the prelimbic region on mediating behaviours by rats. Psychopharmacology, 134, 73-82.

DunNett, S. B., Rogers, D. C., \& Jones, G. H. (1989). Effects of nucleus basalis magnocellularis lesions in rats on delayed matching and nonmatching to position tasks: Disruption of conditional discrimination learning but not of short-term memory. European Journal of Neuroscience, 1, 395-406.

Edhouse, W. V., \& White, K. G. (1988). Sources of proactive interference in animal memory. Journal of Experimental Psychology: Animal Behavior Processes, 14, 56-70.

Elrod, K., Buccafusco, J. J., \& Jackson, W. J. (1988). Nicotine enhances delayed matching-to-sample performance by primates. Life Sciences, 43, 277-287.

Everitt, B. J., \& RobBins, T. W. (1997). Central cholinergic systems and cognition. Annual Review of Psychology, 48, 649-684.

HaRLow, H. F. (1949). The formation of learning sets. Psychological Review, 52, 51-65.

Herremans, A. H. J., Hijzen, T. H., Welborn, P. F. E., Olivier, B., \& Slangen, J. L. (1996). Effects of infusion of cholinergic drugs into the prefrontal cortex area on delayed matching to position performance in the rat. Brain Research, 711, 102-111.

Herzog, H. L., Grant, D. S., \& Roberts, W. A. (1977). Effects of sample duration and spaced repetition upon delayed matching-to-sample in monkeys (Macaca arctoides and Saimiri sciureus). Animal Learning \& Behavior, 5, 347-354.
JENNingS, J. W., \& KeEFer, L. H. (1969). Olfactory learning set in two varieties of domestic rat. Psychological Reports, 24, 3-15.

JoNEs, D. N. C., \& Higgins, G. A. (1995). Effect of scopolamine on visual attention in rats. Psychopharmacology, 120, 142-149.

KoGER, S. M., \& MAIR, R. G. (1994). Comparison of the effects of frontal cortical thalamic lesions on measures of olfactory learning and memory in the rat. Behavioral Neuroscience, 108, 1088-1100.

LANGWORThy, R. A., \& Jennings, J. W. (1972). Odd ball, abstract, olfactory learning in laboratory rats. Psychological Record, 22, 487 490.

Lyford, G. L., Gutnikov, S. A., Clark, A. M., \& Rawlins, J. N. P. (1993). Determinants of non-spatial working memory deficits in rats given intraventricular infusions of the NMDA antagonist AP5. Neuropsychologia, 31, 1079-1098.

MCGregor, I. S. (1996). Using Strawberry Tree WorkbenchMac and Workbench PC software for data acquisition and control in the animal learning laboratory. Behavior Research Methods, Instruments, \& Computers, 28, 38-48.

Muir, J. L., Everitt, B. J., \& RobBins, T. W. (1994). AMPA-induced excitotoxic lesions of the basal forebrain: A significant role for the cortical cholinergic system in attentional function. Journal of Neuroscience, 14, 2313-2326.

Murray, E. A., \& Mishkin, M. (1986). Visual recognition in monkeys following rhinal cortical ablations combined with either amygdalectomy or hippocampectomy. Journal of Neuroscience, 6, 1991-2003.

MurRaY, E. A., \& MishKin, M. (1998). Object recognition and location memory in monkeys with excitotoxic lesions of the amygdala and hippocampus. Journal of Neuroscience, 18, 6568-6582.

Nigrosh, B. J., Slotnick, B. M., \& Nevin, J. A. (1975). Olfactory discrimination, reversal learning, and stimulus control in rats. Journal of Comparative \& Physiological Psychology, 89, 285-294.

Ohno, M., Yamamoto, T., \& WaTanabe, S. (1994). Blockade of hippocampal M1 muscarinic receptors impairs working memory performance of rats. Brain Research, 650, 260-266.

Olton, D. S., Becker, J. T., \& HandelmanN, G. E. (1980). Hippocampal function: Working memory or cognitive mapping? Physiological Psychology, 8, 239-246.

Otro, T., \& Eichenbaum, H. (1992). Complementary roles of the orbital prefrontal cortex and the perirhinal-entorhinal cortices in an odorguided delayed-nonmatching-to-sample task. Behavioral Neuroscience, 106, 762-775.

PONTECORVo, M. J. (1983). Effects of proactive interference on rats' continuous nonmatching-to-sample performance. Animal Learning \& Behavior, 11, 356-366.

Pontecorvo, M. J., Clissold, D. B., White, M. F., \& Ferkany, J. W. (1991). $N$-methyl-D-aspartate antagonists and working memory performance: Comparison with the effects of scopolamine, propranolol, diazepam, and phenylisopropyladenosine. Behavioral Neuroscience, 105, 521-535.

Pontecorvo, M. J., Sahgal, A., \& Steckler, T. (1996). Further developments in the measurement of working memory in rodents. Cognitive Brain Research, 3, 205-213.

Ravel, N., Vigouroux, M., Elaagouby, A., \& Gervais, R. (1992). Scopolamine impairs delayed matching in an olfactory task in rats. Psychopharmacology, 109, 439-443.

Rothblat, L. A., \& HaYes, L. L. (1987). Short-term object recognition memory in the rat: Nonmatching with trial-unique junk stimuli. Behavioral Neuroscience, 101, 587-590.

SAKURAI, Y., \& WENK, G. L. (1990). The interaction of acetylcholinergic and serotonergic neural systems on performance in a continuous nonmatching to sample task. Brain Research, 519, 118-121.

Schellinck, H. M., Brown, R. E., \& SlotNick, B. M. (1991). Training rats to discriminate between the odors of individual conspecifics. Animal Learning \& Behavior, 19, 223-233.

Serby, M., Flicker, C., Rypma, B., Weber, S., Rotrosen, J. P., \& FerRIS, S. H. (1989). Scopolamine and olfactory function. Biological Psychiatry, 28, 79-82.

SLOTNICK, B. M. (1994). The enigma of olfactory learning revisited. Neuroscience, 58, 1-12.

SLOTNICK, B. M., \& KATZ, H. M. (1974). Olfactory learning set formation in rats. Science, $185,796-798$.

Slotnick, B. M., KufEra, A., \& Silberberg, A. M. (1991). Olfactory 
learning and odor memory in the rat. Physiology \& Behavior, 50, 555-561.

Soffie, M., \& LamberTy, Y. (1988). Scopolamine effects on juvenile recognition in rats: Possible interaction with olfactory sensitivity. $B e$ havioral Processes, 17, 181-190.

Spencer, D. G., JR., Pontecorvo, M. J., \& Heise, G. A. (1985). Central cholinergic involvement in working memory: Effects of scopolamine on continuous nonmatching and discrimination performance in the rat. Behavioral Neuroscience, 99, 1049-1065.

Stanhope, K. J., Mclenachan, A. P., \& Dourish, C. T. (1995). Dissociation between cognitive and motor/motivational deficits in the delayed matching to position test: Effects of scopolamine, 8-OH-DPAT and EAA antagonists. Psychopharmacology, 122, 268-280.

Steckler, T., Drinkenburg, W. H. I. M., Sahgal, A., \& Aggleton, J. P. (1998). Recognition memory in rats: I. Concepts and classification. Progress in Neurobiology, 54, 289-311.

VisCardi, A. P., \& Heise, G. A. (1986). Effects of scopolamine on com- ponents of delayed response performance in the rat. Pharmacology, Biochemistry \& Behavior, 25, 633-639.

Voytko, M. L., Olton, D. S., Richardson, R. T., Gorman, L. K., Torin, J. R., \& PRICE, D. L. (1994). Basal forebrain lesions in monkeys disrupt attention but not learning and memory. Journal of Neuroscience, 14, 167-186.

Walsh, T. J., Herzog, C. D., Gandhi, C., Stackman, R. W., \& Wiley, R. G. (1996). Injection of IgG 192-saporin into the medial septum produces cholinergic hypofunction and dose-dependent working memory deficits. Brain Research, 726, 69-79.

Zyzak, D. R., Otto, T., Eichenbaum, H., \& Gallagher, M. (1995). Cognitive decline associated with normal aging in rats: A neuropsychological approach. Learning \& Memory, 2, 1-16.

(Manuscript received September 16, 1998; revision accepted for publication August 12, 1999.) 